\title{
Mobbing Behavior and Deceit and its role in Bio- inspired Autonomous Robotic Agents
}

\author{
Justin Davis and Ronald C. Arkin \\ Mobile Robot Lab, Georgia Tech, Atlanta GA 30332
}

\begin{abstract}
Arabian babblers are highly preyed upon avians living in the Israeli desert. The survival of this species is contingent upon successful predator deterrence known as mobbing. Their ability to successfully defend against larger predators is the inspiration for this research with the goal of employing new models of robotic deception. Using Grafen's Dishonesty Model [3], simulation results are presented, which portend the value of this behavior in military situations.
\end{abstract}

\section{INTRODUCTION}

Mobbing is an anti-predator behavior mainly displayed in cooperative birds but can also be found in animals such as meerkats [13] and squirrels [11] shown in figure 1. This behavior is a prime example of the handicap principle which claims that signals with a high cost must be honest [1]. While this principle is predominantly used for determining when honesty is the most advantageous strategy, it can also be a powerful tool for deciding when deceit is the best option. Some biologists argue that not all signals of this nature are required to be honest.

One such model that incorporates deceit into the handicap principle is Grafen's Dishonesty Model [3], which takes into account several factors to determine whether deception or honesty is the appropriate action to take. Our research has created a model (Sec. 3) based on Grafen's approach. In our research, as part of an ONR MURI ${ }^{1}$ in heterogeneous teams of robots, we replicate situations encountered during the mobbing process and determine when it is advantageous to deceive.

A relatedness coefficient, which serves as a probability of necessity of deception, and a cost associated with the manifestation of this behavior are the primary variables used within the computational model. These parameters can be adjusted with ease to simulate the interaction between the robotic agent and the perceived threat. This new research extends and expands our previous research in deceptive behavior that focused on human models of cognition [7]. In that earlier work, deception was defined simply as a false communication that tends to benefit the communicator (from [8]), and we continue to use that definition in this paper.

One species, often associated with the handicap principle that exhibits this mobbing behavior is the Arabian Babbler. A number of ethological studies have been conducted on

\footnotetext{
${ }^{1}$ This research was supported by the Office of Naval Research under MURI Grant \# N00014-08-1-0696.
}

this bird in Israel [1,2]. The observed behavior of this bird will serve as the inspiration for the robotic simulation that follows. While not all avian species mob in the same manner, general patterns occur and can be applied without the loss of generality.

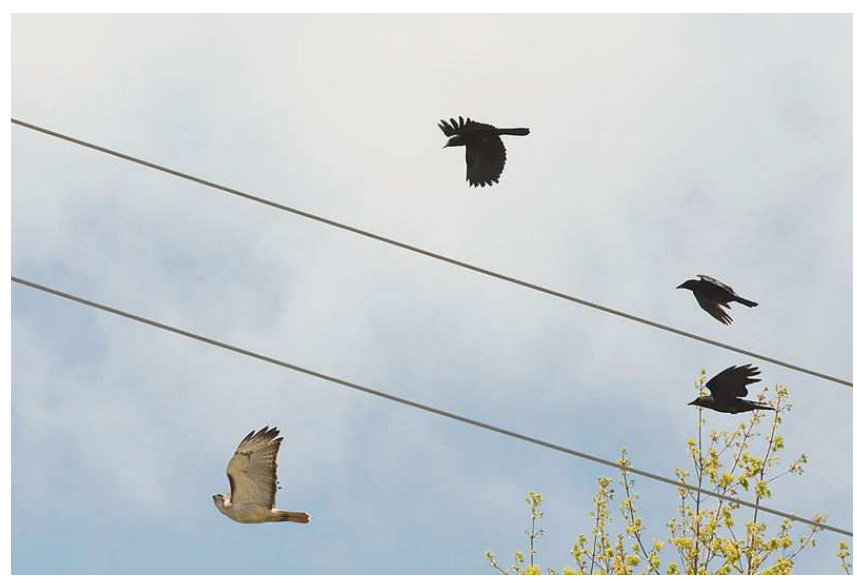

Figure 1: Crows Mobbing a Hawk [15]

In this research we model the mobbing process, most importantly group formation during mobbing, integrate Grafen's dishonesty model, and examine its utility in the context of multiagent robotics. Our preliminary results are provided via simulation studies, with the intent on demonstrating the results in multi-robot experiments in the near future.

The motivation behind this research is for determining when to invoke robotic deception based on principles that transcend individual biological species (namely the handicap principle), specifically in situations when the reward for deceit outweighs the cost of being caught while simultaneously incorporating the cost of sending deceptive signals. This can pertain to military operation. For example, a robot that is threatened might feign the ability to combat adversaries without actually arming the robot: Being honest about the robot's abilities risks capture or destruction while deception could possibly drive away the threat, if used at the right time in the right way. Feigning strength is a tactic used regularly in military combat [9].

\section{MOBBING BEHAVIOR}

In this section, we develop the underlying behavior for the deception scenario within which our model is tested. Mobbing behavior is considered by many biologists to be an altruistic anti-predator behavior. Mobbing is defined as the 
gathering of members of a group around a potentially dangerous individual. The purpose of this behavior is to deter and drive away potential predators. It makes sense that this behavior is only found in animals which are heavily preyed upon. While it is exhibited by many different species, it is most commonly associated in avians. The mobbing birds react to a perceived threat by surrounding it and cooperatively harassing it, usually by making noises and flapping their wings. Other species of birds that mob do so in a similar manner with differences in duration and vocalizations (e.g., ([10]).

A popular example of mobbing is displayed in the Arabian Babbler (Turdoides squamiceps). In babblers, the behavior is determined by group makeup and individual fitness. A group of babblers can consist of anywhere from two to fourteen birds [1]. When a group begins to forage in a feeding area, a single babbler assumes the role of "sentinel" [1]. The sentinel perches in the tree that gives it the best view of its group and approaching predators [2].

The mobbing processes for the sentinel and individual babbler are shown in Figure 2 and 3 respectively. It begins when the sentinel spies a potential danger. It responds by emitting an alarm. Upon hearing this alarm call, individual babblers congregate in the sentinel's tree and assist in issuing these alarm calls. It is suggested that the birds keep making these sounds to let the predator know that it has been seen [1]. If the predator still approaches the group and perches nearby, the babblers approach and mob the predator. During mobbing, the babblers rarely physically attack the intruder, but instead emit vocalizations and circle the predator while flapping their wings. The predator responds by either leaving or attacking one of the mobbing birds. [1].

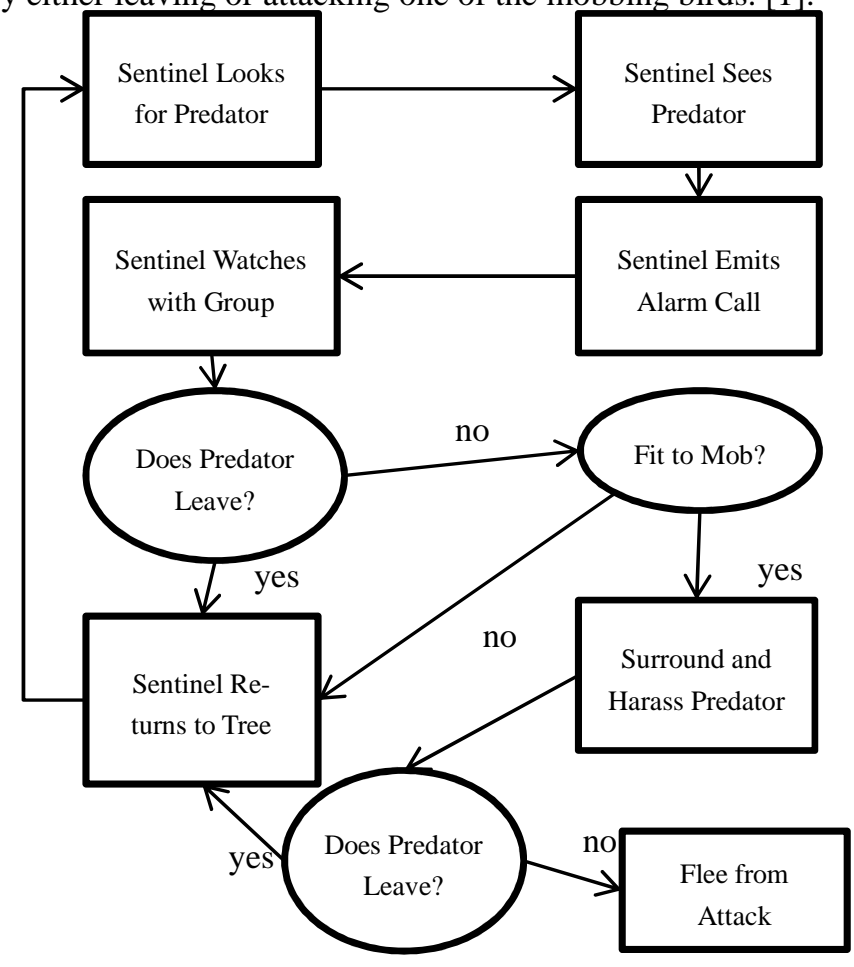

Figure 2: Mobbing Process for Sentinel (based on [1])

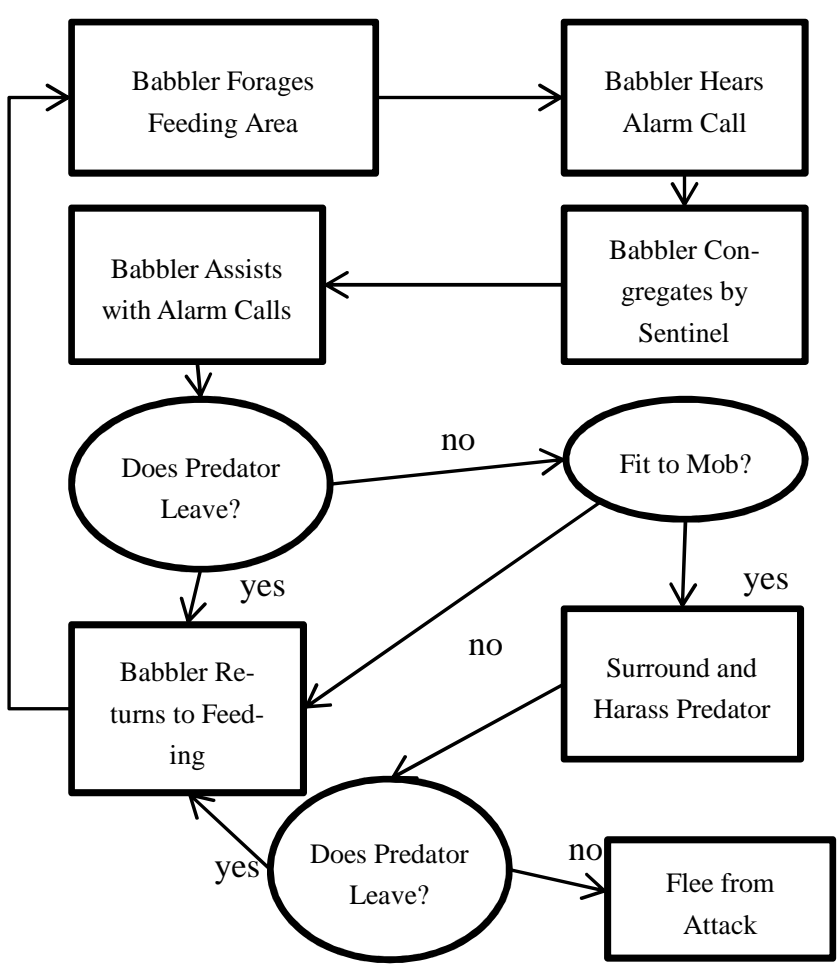

Figure 3: Mobbing Process for Individual Babbler (based on [1])

Not all of the babblers approach the predator. An individual bird's decision to participate in mobbing is influenced by several factors including its rank and its perceived ability to escape. Participation in mobbing can be determined by these factors associated with the group:

- Number of males. In a group containing several adult males, the alpha male generally does not participate in order to stay safe and able to breed in the future. However, if a group has few adult males, the alpha male is more likely to participate.

- $\quad$ Rank. Generally the higher ranking babblers of a group are more active in mobbing. They participate longer and get closer to the predator. According to Zahavi [1], this is to retain their prestige within the group.

- $\quad$ Fitness. Babblers will not approach a predator if they do not feel they are fit enough or possess other means to escape.

While each of these factors is important in determining the flock's decision to mob, the work described here focuses on an individual babbler's perceived ability to escape and is the focus of our implementation. This is the part of the mobbing process where "deception" will be injected into the system. Here, a babbler may deceive regarding its fitness in order to deter the predator. While there is no direct evidence to suggest this occurs in nature, it presents an interesting variation for robotic decisions regarding feigning behavior.

It is important to view mobbing as a signal between the prey and the predator. Thus, a simple signal sent between the two agents is sufficient to model this behavior. While the simulation presented shows the babblers harass- 
ing the predator, the display itself does not determine the predator's behavior at this step. This model incorporates the ability for the prey to "deceive" the predator. Using the model, one could determine an appropriate time to feign strength to the adversary or to conserve resources in escaping in a military scenario.

\section{B. Sentinel}

The catalyst to the mobbing process is the sentinel issuing an alarm call. The role of sentinel is assumed by a member of the group [1] and is usually filled by the alpha male or another high-ranking male. For each group of babblers, there is only one sentinel at any given time. In a natural setting, the sentinels change, but for the purposes of this simulation, the sentinel will be predetermined and static. The sentinel, like other birds in the group, participates in mobbing with respect to Grafen's Dishonesty Model [3].

\section{Handicap Principle}

The Handicap Principle, developed by Zahavi [1], details the criteria in which signals between animals are required to be honest. It states that if an animal wastes its personal resources to produce a signal, then that signal must be honest. Otherwise, it cannot afford to waste such resources. This is a fairly accepted principle now but was highly contested when it was first introduced. Its application to mobbing is that babblers will not approach a predator if they do not believe they can escape it. If the babbler does approach, it is wasting the resources of cover from the trees and a head start to escape from the predator. By wasting these resources, it is demonstrating that it can survive without them and thus signaling to the predator that a chase is pointless. If it could not tolerate losing these resources and attempts to mob the predator anyway, that babbler becomes vulnerable to an attack. In that case, the babbler would not be able to survive should the predator decide to attack it, and thus deceiving with respect to its low fitness was not the appropriate choice according to the handicap principle.

\section{Deception}

The purpose of this research is to model the mobbing behavior and determine what value it affords robots and what, if any, value is added by injecting deception into the process. Deception in this case is what biologists describe as cheating [3]. While Zahavi maintains that signals produced through wasted resources must be honest, Grafen claims there can exist an acceptable level of cheating that will keep the system stable [3]. Grafen details inequalities in which cheating would be the best strategy for the signaler. The derived model is based upon the "Philip Sydney game" [3]. In this situation, cheating constitutes a babbler signaling to the predator that it can escape any subsequent chase when it actually could not. If a predator attacks a babbler that is bluffing about its fitness, the babbler will most likely be captured and eaten, a rather serious gamble.

\section{E. The Philip Sydney Game}

The Philip Sydney game is a signaling game between two players, developed by John Maynard Smith [3], which we will consider in the context of predator-prey relations. The two players in the game are a donor and a beneficiary. The donor has a resource that the beneficiary may or may not need, e.g., water. The beneficiary has the ability to signal to the donor that it does or does not need this resource. Upon receiving this signal, the donor can decide whether or not to give the resource to the beneficiary. Several factors go into the decision as to whether or not the beneficiary should signal that it needs the resource including a relatedness coefficient and a necessity coefficient. Similar parameters go into the decision for the donor to give up the resource [3]. There are a few different outcomes of all these decisions. In the example of the resource being water, if the donor gives up the water, there is a possibility that it will not survive due to thirst. On the other hand if the donor keeps the water, there is chance that the beneficiary perishes. If the beneficiary signals, it pays a cost to its fitness and upon not receiving the water, maintains a lower survival rate. Thus it is very important to for the beneficiary to signal appropriately. For the scenario we consider, the donor is the predator, the beneficiary is the babbler, and the resource offered is the predator sparing the babbler's life. A more detailed description of the model appears in section 3 .

\section{F. Group Control}

Mob formation does not have an exact spatial layout and positioning as was the case in our earlier work on formations [Balch and Arkin 98], but some spatial constraints define the mob structure. For example, the babblers that are mobbing must space themselves out around the predator. In earlier work [5], bird lekking behavior was used for group formation in a different context, that of trying to find and attract a scarce resource. Utilizing this pre-existing group formation behavior is an easy solution for implementation. In lek behavior, all group members are attracted to a hotspot (location where resources are likely to be found) but modestly repelled by other members to assure a uniform spatial distribution. For mobbing these roles are altered: the predator settles itself at the hotspot, where the hotspot in this case is the perching location of the predator around which the other babblers group during the mob.

\section{COMPUTATIONAL MODEL}

\section{A. Sentinel Behavior}

The computational model for the sentinel behavior is shown in figure 4, and is derived from the behavioral processes shown in figure 2. Each component behavioral assemblage (an aggregation of primitive behaviors [14]) and their associated transitions (behavioral triggers) are described below. 


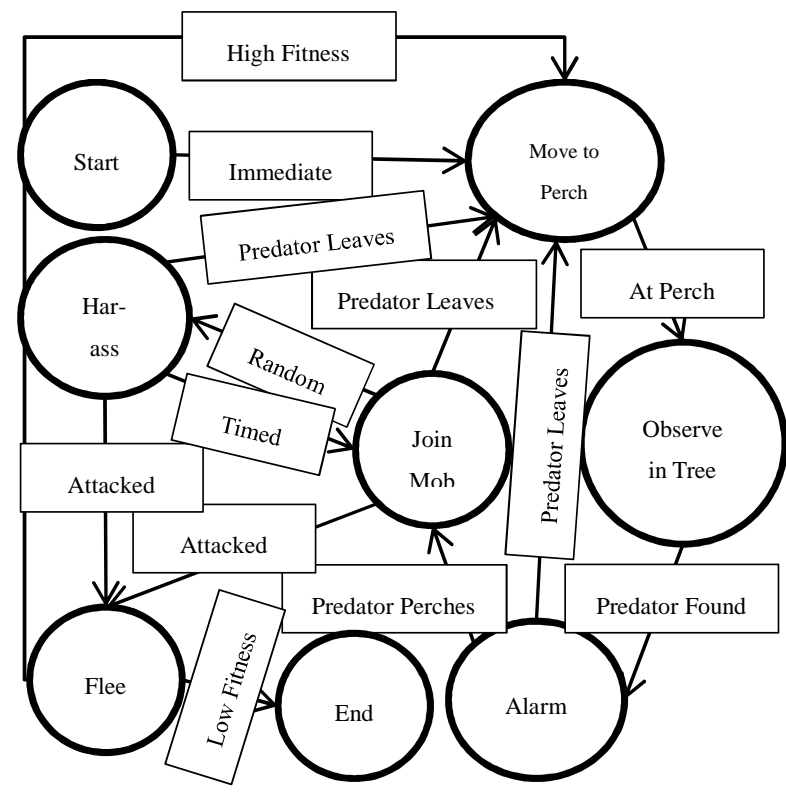

Figure 4: Computational Model for Sentinel Behavior

\section{$\underline{1 . \text { Move to Perch }}$}

The sentinel begins by moving to the perch area. This will be a static location where it can best observe and detect predators threatening it and other group members. This behavioral assemblage is composed of the primitive Move_To behavior combined with obstacle avoidance. The sentinel transitions when it reaches the perching area to observing. Based upon sentinel behavior described in [2], the specific perching area chosen gives the sentinel the best view. Thus no visual occlusions due to obstructions are assumed since the bird naturally chooses a spot that likely does not contain such impairments.

\section{Observe in Tree}

In this behavioral state the sentinel remains stationary while attempting to detect a predator. Upon detection of a predator, a transition occurs to the Alarm state. According to [6], European Starlings (Sturnus Vulgaris) can detect a predator $40 \mathrm{~m}$ away and detect them by sight. These birds have similar physical characteristics to Arabian babblers and thus this provides the detection distance used in the simulation.

\section{Alarm}

The alarm assemblage the sentinel notifies both allies and enemies that an enemy has been detected. Simultaneously the sentinel broadcasts its location to both friendly and enemy agents. The enemy is notified because the sentinel is giving up its position in order to let the predator know it has been seen. This is in agreement with the handicap principle [1] as the babbler is wasting its advantage of being hidden in order to send the signal. The behavioral make-up of this assemblage requires the sentinel to notify both the babblers and the predator of its current position so that the non-sentinel babblers can move toward it for mobbing and so the predator knows it has been seen. If the predator leaves, the sentinel remains in its perch. If the predator lands, the sentinel will mob the predator. This assumption is made since the sentinel always has the required fitness to mob the predator.

\section{Join Mob}

When in this state the sentinel moves towards the predator, while employing obstacle avoidance to avoid collisions with trees and other birds. When mobbing, the simulated babbler agents are attracted to the predator while maintaining a certain distance from it. This area is known as the attraction zone [5]. There is also a radius around the predator where the mobbing agents will be repelled. This is the repulsion zone [5]. Attraction and repulsion to the predator is detailed in equation 1.

$$
V_{\text {mag }}=\frac{H-d}{H}
$$

here $V_{\text {direction }}=$ Direction from the center of the robot to the center of the predator, $\mathrm{d}=$ Distance of robot to predator, and $\mathrm{H}=$ Maximum predator detection distance.

When mobbing the predator, the mobbing agents space themselves evenly around the attacker dictated by equation 2:

$$
V_{\text {mag }}\left\{\begin{array}{cc}
\frac{R-d}{R}, & R \leq d \\
0, & A_{\min }<d<R \\
\frac{A_{\max }-d}{A_{\max }-A_{\min }} & A_{\max } \leq d \leq A_{\min }
\end{array}\right\}
$$

where $V_{\text {direction }}=$ Direction from the center of the robot to the center of the other robot, $d=$ Distance of robot to another robot, $\mathrm{R}=$ Radius of the repulsion sphere, $\mathrm{A}_{\min }=$ Inner radius of the attraction sphere, and $\mathrm{A}_{\max }=$ Outer radius of the attraction sphere. This model was introduced in our earlier work on bird lekking behavior, which also includes obstacle avoidance. [5].

Noise is also introduced into the system to assist in avoiding local minima. Transition out of the Join_Mob state to the Harass state occurs probabilistically. The probability should ideally be determined empirically through field studies to reproduce what occurs in nature. That data, however, is not currently available so an arbitrary value is chosen. During the Join_Mob state, the prey agents surround the predator. If the predator leaves, the sentinel transitions to the Move_to_Perch state, returning to its previous observation point. If the sentinel is attacked by the predator it will flee. An agent that is attacked is destroyed if its fitness is too low for survival. If the sentinel survives mobbing the predator, it returns to looking for predators (Observe in tree state) via the Move_to_Perch state.

\section{Harass}

When in the Harass state, the sentinel moves toward the predator causing the predator to become frustrated. Upon repeated harassing if the frustration level of the predator becomes sufficiently high the predator will leave. Otherwise the harasser returns to the mob after a given time, unless the 
predator leaves or attacks while harassing. In the first instance, the sentinel returns to the perch, but if attacked it flees as before.

\section{B. Non-Sentinel (Individual) Behavior}

The model for the individual babbler agent behavior is shown in figure 5 . This overlaps considerably with the Sentinel model with the biggest differences being the absence of a Feed state in the sentinel, and the individual babbler does not contain an Alarm state. The triggers between most assemblages are also slightly different. These differences are explained below.

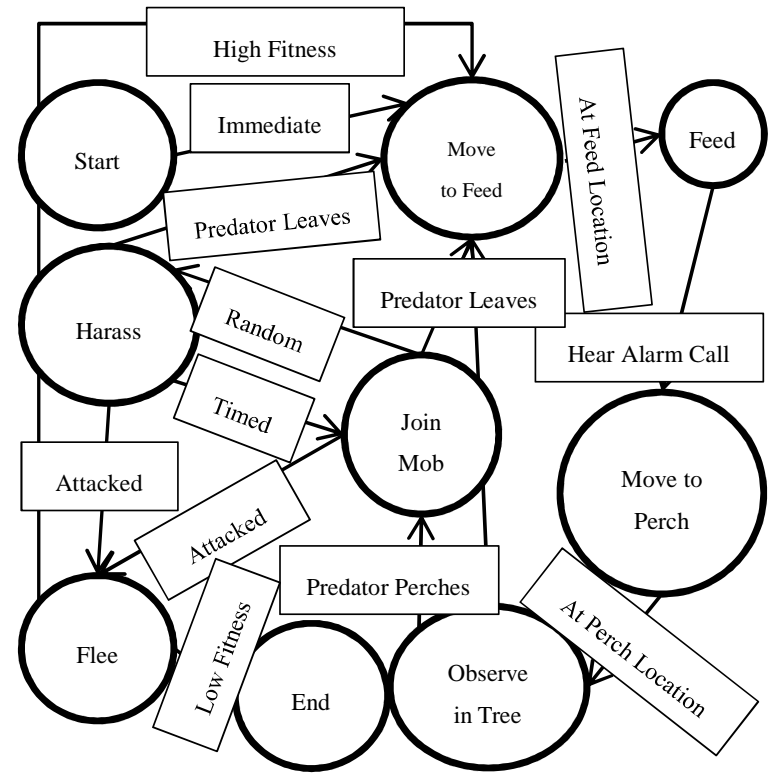

Figure 5: Computational Model for the Individual Babbler

\section{Move to Feed}

When the simulation starts, the non-sentinel individuals move to their feeding location. This area is near the sentinel perching location. Upon arrival they enter the feed state.

\section{Feed}

When feeding, the individuals stay at the feeding location until the sentinel emits the alarm call. The alarm serves as a signal indicating the presence of a predator.

\section{Move to Perch (non-sentinel)}

This behavioral assemblage is identical to the sentinel's Move_To_Perch state with the exception that the individuals move to the broadcasted position near the predator determined from the alarm. Upon arrival in the tree with the sentinel, they observe the predator.

\section{Observe in Tree (non-sentinel)}

This assemblage is identical to the sentinel's, and transitions in the same manner. The transition from this state is contingent upon the predator perching and the babbler deciding to join the mob. Equation 3 is derived from Alan
Grafen's dishonest model [3]. A difference In this case the signal cost is added instead of being subtracted because a higher signaling cost should lessen the probability that an individual would mob. If the cost was subtracted, as shown in [3] then the opposite effect would occur. If the inequality shown in equation 3 is met then the babbler joins the others in mobbing.

$$
\begin{gathered}
X=1+t+2 r\left(S_{d}-1\right) \\
M o b\left\{\begin{array}{cc}
Y e s & S_{b}>X \\
N o & S_{b} \leq X
\end{array}\right\}
\end{gathered}
$$

where $S_{b}$ is the individual babbler's fitness, $S_{d}$ is the predator's fitness as perceived by the individual babbler, $r$ is relatedness coefficient, and $t$ is cost of signaling. $X$ represents the risk associated with mobbing this predator. The bounds of all parameters presented, with the exception of $\mathrm{X}$, are 0 and 1 . It is important to note that the parameter $S_{d}$ represents perceived fitness rather than the actual fitness, which will be represented differently in the data analysis. If the inequality is not satisfied, the individual remains in the tree until the predator leaves. An explanation of the validity of this is model can be found at the end of this section.

\section{Join Mob (non-sentinel)}

If the babbler mobs, it will surround the predator in the exact same manner as the sentinel does. Identically to the sentinel model, there is a random chance that the individual will harass the predator. If the predator leaves, a transition will be made back to the Move_to_Feed state rather than moving to the perch location as described in the sentinel behavior.

\section{Harass (non-sentinel)}

The harass state for the individual babbler is identical to the harass state of the sentinel except upon the predator a leaving the agent returns to feeding.

\section{Predator}

The current predator model is simplistic and has no decision-making abilities. It always moves towards the group, perches near the group, and attacks the group after a specific amount of time or leaves because frustration built up due to mobbing agents. In our near-term plans, the predator will have a range of choices regarding when to attack and other aspects of the prey-predator relationship [1], but this paper centers on the mobbing behavior itself.

\section{Deception in Mobbing}

Dishonesty is incorporated into the computational model (after [3]) and is used when the individual makes the choice whether to participate in mobbing or not. If the system was entirely honest then the only factors involved in mobbing would be the fitness of the predator and prey and the cost of the signaling. In the honest situation, if the individual has fitness greater than the predator after factoring in signaling cost, then it would always mob. Similarly, if the individual was fitness deficient after subtracting the signal cost, then it 
would never participate in the completely honest situation. Essentially this states that bluffing or feigning strength is never allowed.

However, when incorporating deception a relatedness coefficient is included, which allows and influences deceptive behavior. This dishonesty model at first glance is not intuitive, requiring a closer look to make apparent its intent. The purpose is to determine when it is the most appropriate strategy for an agent to engage in mobbing independent of whether it is an honest or dishonest signal. Equation 3 assesses the risk of being attacked and devoured by the predator. The higher the risk, the less likely the babbler will mob. All parameters affect the risk according to their influence as explained below.

In equation 3, as signal cost, $t$, increases, the overall fitness of the prey decreases [1]. Thus, $t$ is added to the right side of the equation, increasing risk parameter $\mathrm{X}$, and decreasing the likelihood that mobbing occurs. The model is contingent upon the prey noticing the predator approaching [1]. If the predator is not detected by the prey, this model does not apply. However, once observed by the prey, the risk of the predator attacking increases as the fitness of the predator increases, as reflected in equation 3. It is assumed that a fitter predator has a greater chance of catching prey than a less fit one. For this reason, the predator's fitness, $S_{d}$, is added, indicating it is riskier for a prey individual to approach this predator. The predator's fitness value is subtracted by one, and this quantity is then multiplied by the relatedness coefficient, $r$.

The relatedness coefficient, $r$, drives the decision to mob and expresses the cooperation between predator and prey. The prey does not want to be chased, and the predator wants an easy meal and to not waste energy during a chase [1]. The prey cooperates by telling the predator is has been detected and should move on. The predator cooperates by moving on and not attacking. As $r$ increases, the agents are more likely to cooperate, the risk of being attacked decreases, and the chance of mobbing should increase. This is in agreement with equation 3 , because predator fitness, $S_{d}$, is between 0 and 1 . Subtracting 1 from $S_{d}$, as performed in equation 3, means that $r$ will be multiplied by a negative quantity, implying an inversely proportional relationship. Figure 6 shows the relationship of each parameter assuming a linear model.

Figure 6 shows that $t$ and $S_{d}$ are proportional while $r$ is inversely proportional to $\mathrm{X}$. The dotted line through the center represents $S_{d}=0.6$. Clearly from the figure, a low value of $t$ is necessary for mob participation to occur, which is in agreement with Zahavi [1].

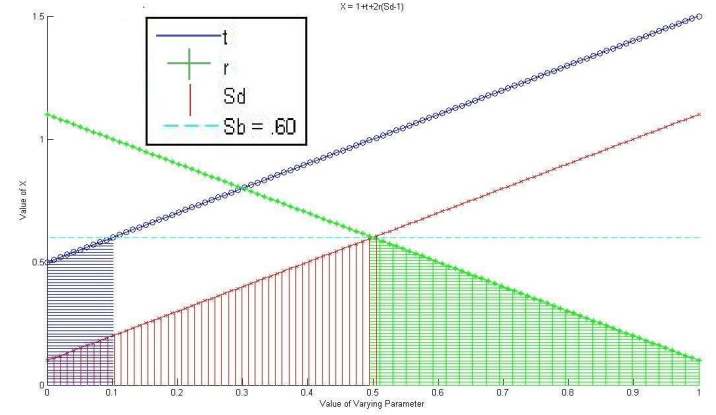

Figure 6: Relationship between input parameters to the model. Generated by holding 2 parameters constant and assigning values between 0 and 1 for the third parameter and seeing the effect. Constant values used were $t=.1, r=.75$ and $S_{d}=.5$. The textured regions represent areas where the individual participates. Each texture corresponds to a different parameter denoted by the legend. The figure shows that parameters $t$ and $S_{d}$ have a proportional relationship with $X$ while $r$ maintains an inversely proportional relationship with $X$.

\section{IMPLEMENTATION}

The computational model has been implemented in MissionLab ${ }^{2}$, software developed by the Mobile Robotics Lab at Georgia Tech. This software allows both for simulation and robotic implementation and supports multiple robotic platforms. This specific research has only utilized the simulation aspect thus far but we expect to port this model to Pioneer robots in the near future.

The implementation of mob behavior is constructed from multiple previously developed behaviors (Appendices A-C). Mob formation around the predator is emulated using a subFSA containing the lek behavior [5]. For the harassment aspect of mobbing, the change-color behavior is utilized rather than implementing any extravagant motor display. The color change (to green) indicates to the predator that the prey agent is in the harass state. The transition between the mob and harass state is probabilistic. This value is empirically assigned, as we have not found supporting biological data regarding the frequency of harassment during mobbing. After harassing is complete, its color returns to the original state, and the agent is considered back in the mob state.

When being harassed, the predator detects green harassers. During each time cycle, the predator's current frustration value is incremented by 1 for each harassing agent:

$$
f_{\text {current }}=f_{\text {previous }}+n
$$

where $f_{\text {current }}$ is the current frustration value, $f_{\text {previous }}$ is the previous frustration value, and $\mathrm{n}$ is the number of harassing agents in a fixed time cycle. If the frustration value exceeds a specified frustration threshold $\left(f_{t}=100,125\right.$, or 150 for

2 MissionLab is freely available for research and educational purposes at: http://www.cc.gatech.edu/ai/robot-lab/research/MissionLab/ 
different simulation runs), the predator leaves and the simulation terminates. If, however, $t$ time cycles elapse (in this case arbitrarily 10) and the frustration threshold has not been exceeded, the predator selects a random mobbing prey individual to attack. If the predator has a higher perceived fitness value than the prey individual it selects, then that agent is considered to be bluffing, and the probability of that agent being killed, $\mathrm{D}_{1}$, is $95 \%$. Conversely if the predator selects an honest mobbing agent, the probability of this agent being killed, $\mathrm{D}_{\mathrm{h}}$, is set to either $5 \%, 10 \%$, or $15 \%$. The chance of the predator killing an honest mobber is increased across different analyses to represent the effect of a fitter predator. Aside from the mob behavior, all communication between robots is through message passing and is used to coordinate state transitions.

The most significant addition regarding deception was the creation of the trigger that decides when to mob and is based on equation 3. Each non-sentinel prey evaluates this inequality whenever in the presence of a predator. Every agent that satisfies this inequality participates in mobbing upon receiving the alarm call from the sentinel. Group members that do not mob do not factor into the simulation's effect on the predator's frustration level or resulting mortality rates. In the results that follow, parameters $t, r$, and $S_{d}$ are held constant $\left(t=0.1, r=0.75, S_{d}=.5\right)$ while the parameters $\mathrm{S}_{\mathrm{b}}$ (fitness), $\mathrm{f}_{\mathrm{t}}$ (frustration threshold), and $\mathrm{D}_{\mathrm{h}}$ (Death probability for honest agents) vary. The assigned value for $S_{b}$ was either $0,0.4$, or 0.6 . A fitness value of 0 indicated the babbler did not participate in mobbing, 0.4 indicated participation in mobbing as a deceptive participant, and 0.6 represented an honest mobbing babbler. All combinations of honest and dishonest mob groups were analyzed for group sizes of 2 through 7 babblers. $f_{t}$ ranged from 100, 125, or 150 with increasing values representing a more patient predator. Finally, the probability of an honest mobber being killed was varied from $5 \%, 10 \%$, and $15 \%$. As previously mentioned, there is a difference between the perceived fitness, $S_{d}$, and the actual fitness. Varying $\mathrm{D}_{\mathrm{h}}$ represents changing actual fitness. Using the assumption that fitter predators are more likely to catch prey, increases in $\mathrm{D}_{\mathrm{h}}$ indicate increases in actual predator fitness. This is more desirable than changing $S_{d}$ as changing perceived fitness alters the number of mobbing agents.

\section{SIMULATION RESULTS}

Figure 7 illustrates an exemplar simulation run at several stages for a group of 4 babbler agents. Initially, the agents are in the feeding state while the sentinel is looking for a predator (Fig. 7A). When the sentinel sees a faraway predator, the other group members congregate as shown (Fig. 7B). All prey agents that have decided to participate in mobbing based on the model described earlier change their color to black at this step. The agents that do not meet the requirements to mob, remain blue. In order to avoid interference in formation around the predator, all non-mobbing agents localize far away from the predator perching area (Fig. 7C). Results of formation around the predator are identical to the earlier lekking results [5]. The predator is then harassed randomly as previously described (Fig. 7D) until it leaves (Fig. 7E) or attacks.

The simulation data was analyzed for the aforementioned values of parameters $S_{b}, f_{t}$, and $D_{h}$. Figures $8 \mathrm{a}, 8 \mathrm{~b}$, and $8 \mathrm{c}$ show the mortality rate for each combination of mob sizes and deception rates present in the group, when $\mathrm{D}_{\mathrm{h}}$ was held constant at .05; while $\mathrm{f}_{\mathrm{t}}=100$ in $8 \mathrm{a}, 125$ in $8 \mathrm{~b}$, and 150 in 8c. Figures $9 \mathrm{a}, 9 \mathrm{~b}$, and 9c demonstrate the same combinations but where $D_{h}=.10$, and figures $10 \mathrm{a}, 10 \mathrm{~b}$, and $10 \mathrm{c}$ show this data when $\mathrm{D}_{\mathrm{h}}=.15$.

For each frustration threshold, there exists a minimum number of mobbing agents $\left(\mathrm{M}_{\mathrm{m}}\right)$, for which the predator's frustration always exceeded its $f_{t}$ and fled. The minimum number of mobbers for which zero attacks occur across each $f_{t}$ is shown in table 1 . Attacks being reduced to 0 results in a $0 \%$ mortality rate. Intuitively this means that lying to create a mob group of this size results in no deaths as reflected in each figure. However, deceiving in groups smaller than these minimum mob sizes is lethal. The deadliest conditions for lying, when $\mathrm{f}_{\mathrm{t}}=125$ and 150 , was a mob formation consisting of 2 deceiving agents and a sentinel. Mobbing a predator with these frustration thresholds and only deceiving agents, resulted in a mortality rate of approximately $70 \%$. It is not surprising that this is the worst condition because $66 \%$ of the group is deceiving while an attack occurs $100 \%$ of the time. Similarly we could expect mortality rate to increase for larger groups with $100 \%$ deception in non-sentinels given a predator with an increased frustration threshold. When $\mathrm{f}_{\mathrm{t}}=100$, the highest mortality rate occurs when 1 deceiving agent and a sentinel participate.

It is desirable to discover if adding deceiving agents to a purely honest situation would result in fewer fatalities. Obviously when adding enough deceivers to exceed or equal $\mathrm{M}_{\mathrm{m}}$ for each frustration threshold value, the mortality rate drops to zero. However it is more interesting to investigate critical mob sizes $\left(M_{c}\right)$ that can result in both the predator attacking or fleeing. $M_{c}$ for each frustration value is presented in table 1 . The surface plots (Figs 8-10) show that a purely honest mob group has a higher survival rate than any group containing a deceiver, with two exceptions. As evidenced in figure $9 b\left(f_{t}=125 D_{h}=10\right)$, a group of 3 honest mobbers yields a mortality rate of .16. Adding one deceiving babbler to this group reduces the mortality rate by $25 \%$. Similarly, as seen in figure $10 \mathrm{c}\left(\mathrm{f}_{\mathrm{t}}=150 \mathrm{D}_{\mathrm{h}}=.15\right), 3$ honest mobbing babblers have a mortality rate of 0.20 . Adding one deceiving babbler drops the mortality rate by $30 \%$. 


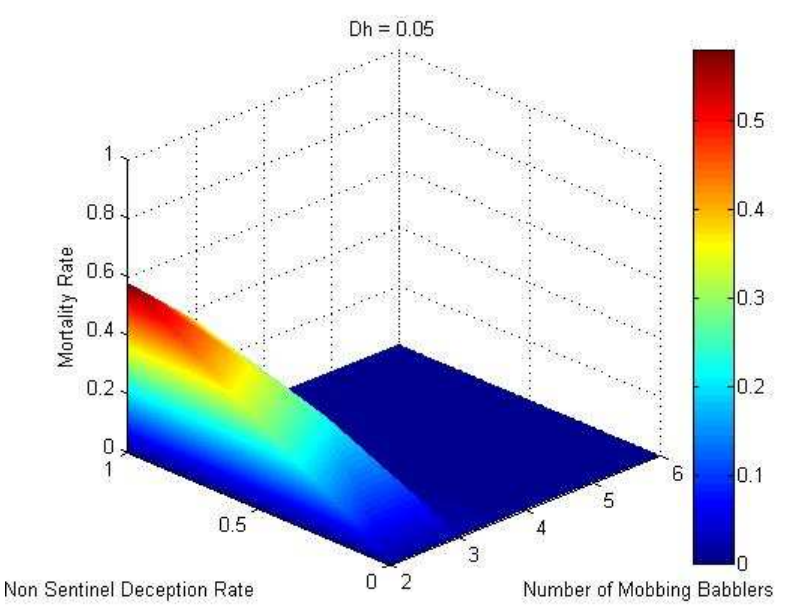

Figure 8a-c: Surface plot of number of babblers, deception rate in group member which are not the sentinel, and the mortality rate from the group makeup. The sentinel is always an honest mobber. Each subsequent plot contains different values for the parameters $f_{t}$ and $D_{h}$. For all plots, the probability that a lying agent is killed, $D_{l}$, is $95 \%$. For this plot $f_{t}=100$ and $D_{h}=.05$.

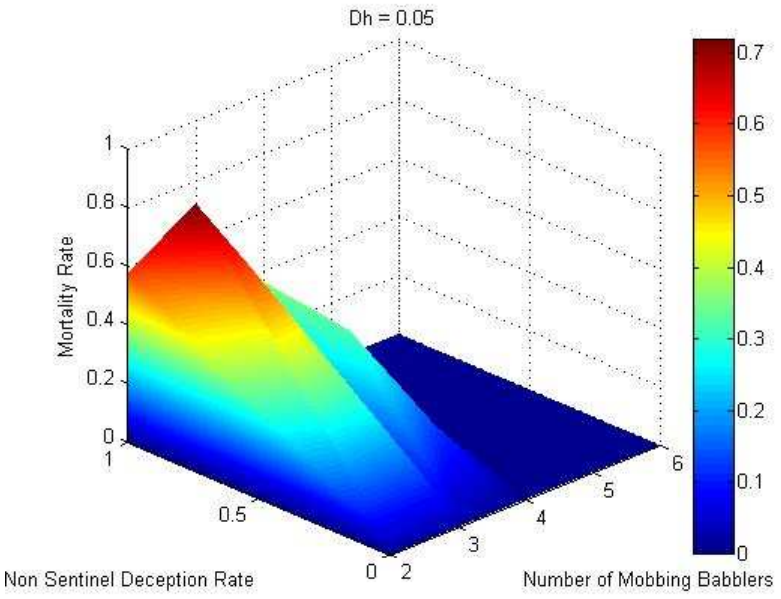

Figure $8 b: f_{t}=125 D_{h}=.05$

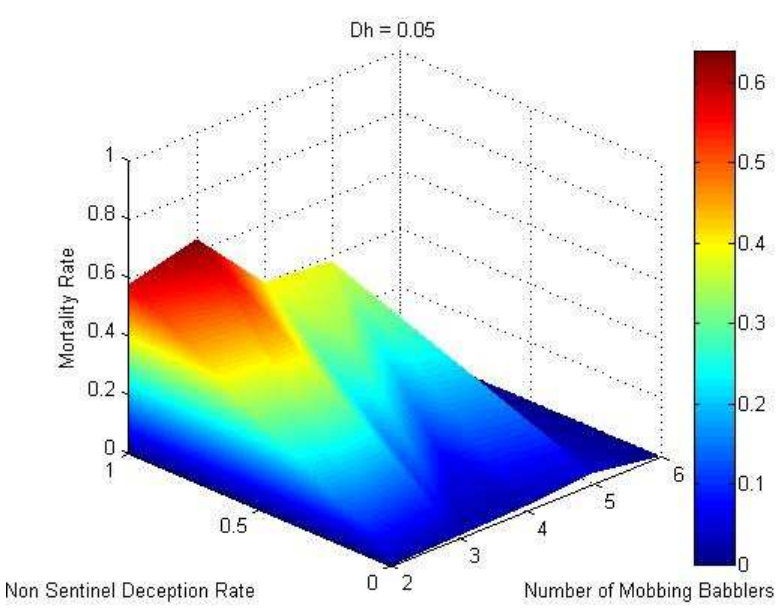

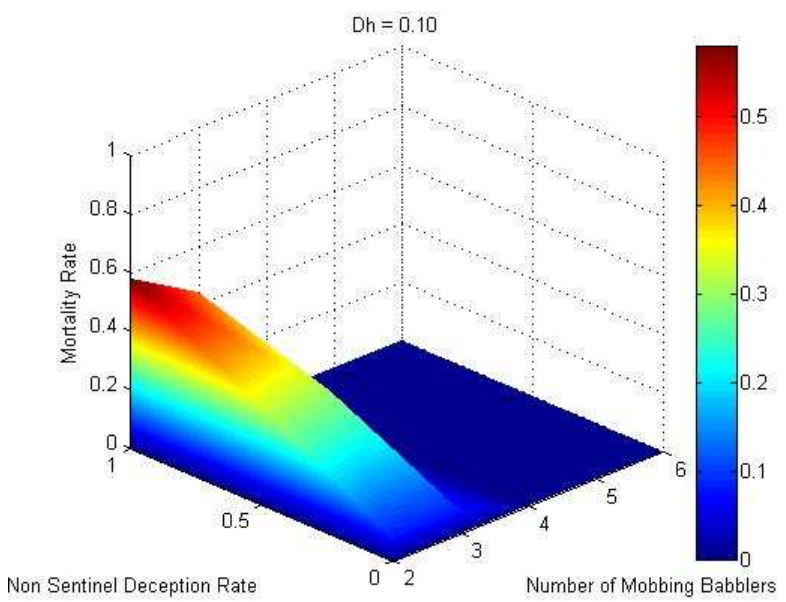

Figure 9a: $f_{t}=100 D_{h}=.10$

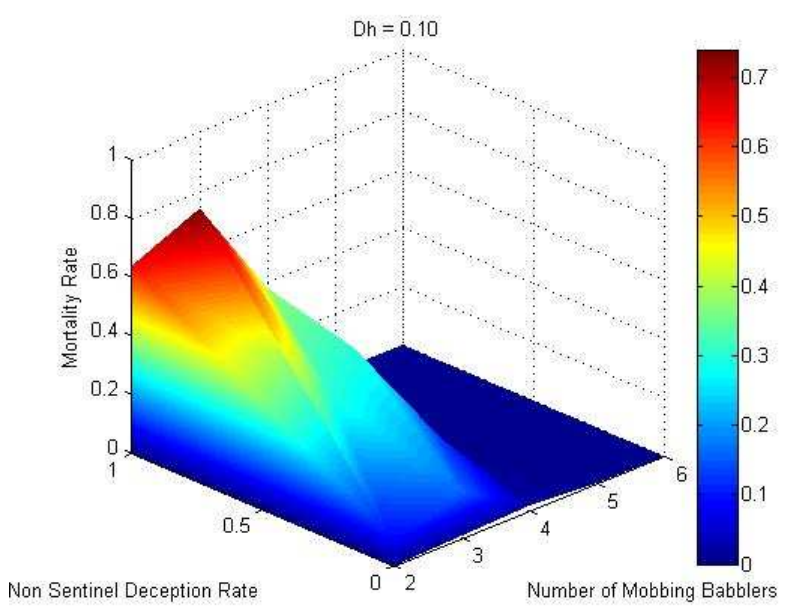

Figure $9 b: f_{t}=125 D_{h}=.10$

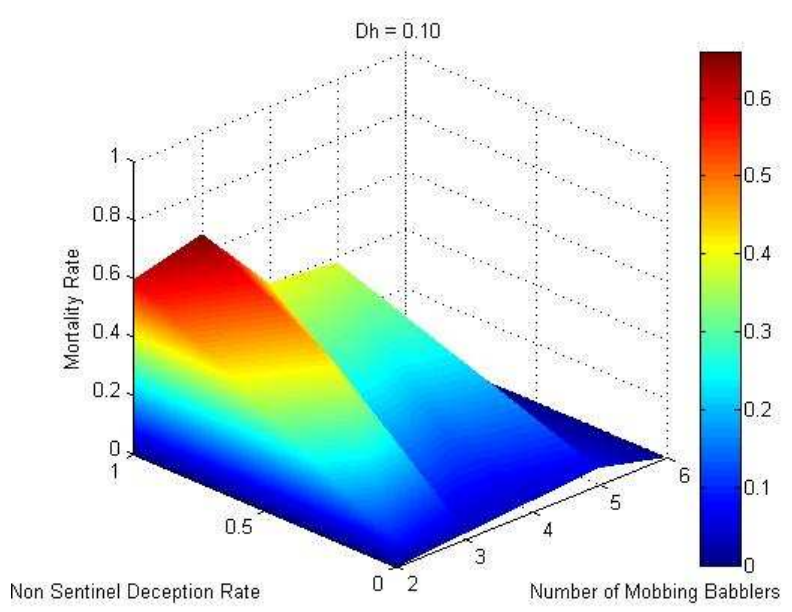

Figure $9 c: f_{t}=150 D_{h}=.10$

Figure $8 c: f_{t}=150 D_{h}=.05$ 


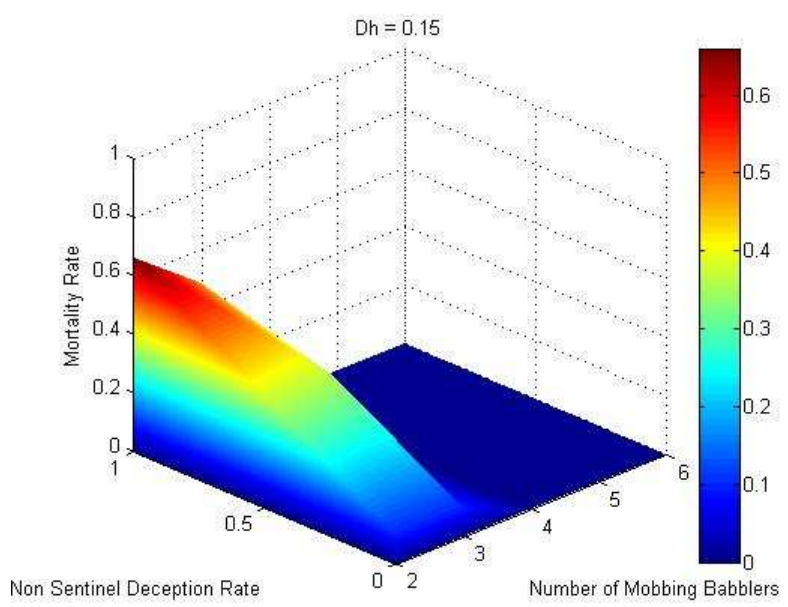

Figure 10a: $f_{t}=100 D_{h}=.15$

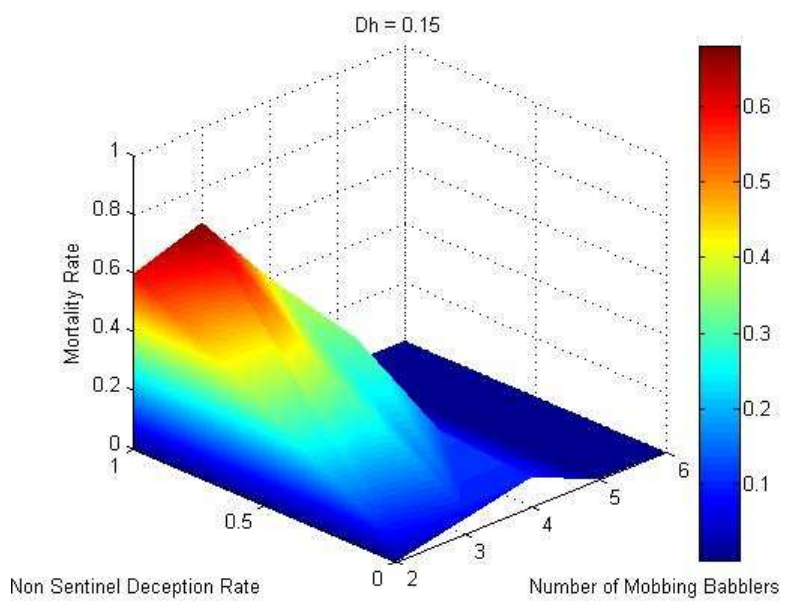

Figure 10b: $f_{t}=125 D_{h}=.15$

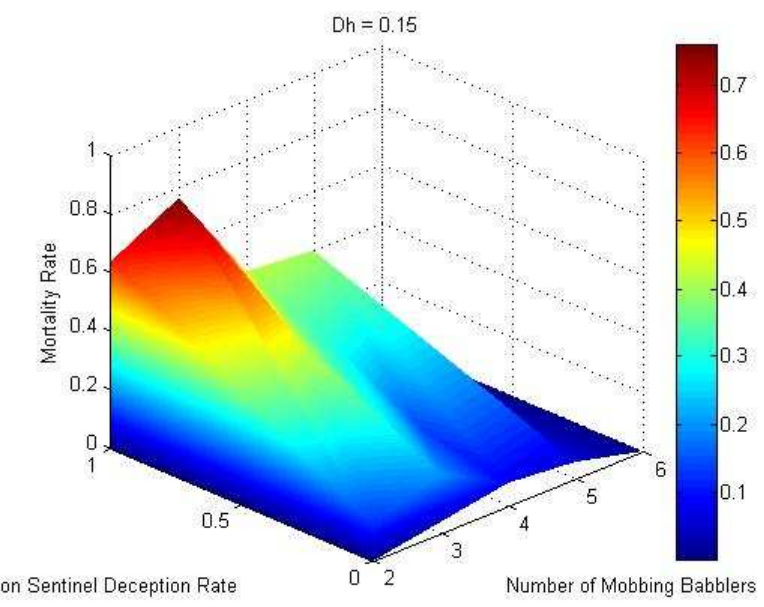

Figure 10c: $f_{t}=150 D_{h}=.15$
Table 1: Minimum Mob Sizes for 0 attacks and Mob sizes where the predator attacks and flees for each frustration threshold

\begin{tabular}{|c|c|c|}
\hline $\mathrm{f}_{\mathrm{t}}$ & $\mathrm{M}_{\mathrm{m}}$ & $\mathrm{M}_{\mathrm{c}}$ \\
\hline 100 & 4 & 3 \\
\hline 125 & 5 & 4 \\
\hline 150 & 6 & 4,5 \\
\hline
\end{tabular}

Since these are the only two incidents in the entire data set in which the addition of a single deceiver decreases the mortality rate, it can be concluded that lying with $\mathrm{D}_{1}=.95$, is not a strategic decision in mob groups less than $\mathrm{M}_{\mathrm{m}}$. Figure 11 shows the result of reducing $\mathrm{D}_{1}$ to $50 \%$ and increasing $D_{h}$ to $30 \%$ while $f_{t}$ was 150 . Under these new conditions, deception improves survivability in group sizes of $\mathbf{M}_{\mathrm{c}}$. Adding one deceiving member to mob size of 3 with any deception rate decreased the mortality rate by an average of $16 \%$. While this may not be realistic, it proves that there is a set of conditions in which deceiving can improve survival rate consistently. In order for this to occur, the penalty for deception must be low. This is in accordance with [3] in which Grafen stated that lying is only tolerated in systems where its cost is small.

In summary, when $D_{l}$ is high and $D_{h}$ is low, the role of deception is to increase the mob size to exceed or equal $M_{m}$, which in turn drops the mortality rate to 0 . When $D_{1}$ decreases and $D_{h}$ increases, deception can be used to drop the mortality rate when the mob size is $\mathbf{M}_{\mathrm{c}}$.

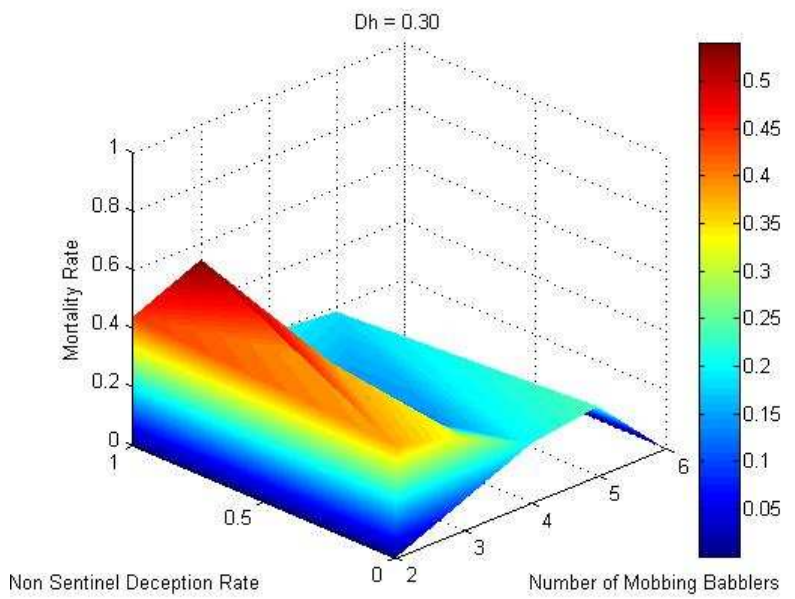

Figure 11: In this figure, $D_{h}$ has been increased to .30 and $D_{l}$ has been reduced to .50 . While this may not reflect nature, it shows a set of data in which deception can help to reduce the mortality rate.

\section{CONCLUSION}

Mobbing behavior, in nature, has clearly proven to be an effective method of predator deterrence. Our research shows the ability to transfer this biologically-inspired behavior to robotic behavior in simulation, where the robotic 
agents emulate the ethology of mobbing birds. The addition of deception to the handicap principle returns mixed results.

For this simulation, deception is the best strategy when adding a single agent pushes the mob size to $\mathbf{M}_{\mathrm{m}}$. In this case, the predator is driven away and no member is attacked. For mob sizes smaller than $\mathrm{M}_{\mathrm{m}}$, complete honesty yields the smallest mortality rate. This is because the punishment for bluffing is high. If the price of bluffing is reduced, adding deception can result in a reduced mortality rate when the predator attacks.

Mobbing, based on our earlier implementation of bird lekking [Duncan et al 09], permits the agents working in teams to create multiple formations and allows them to group in specific areas. Future work will investigate the impact of varying $\mathrm{D}_{1}$ on mortality rate and implementing this simulation on physical robotic systems, specifically Pioneer robots as we have done in previous research on lekking. Understanding how many honest and dishonest prey agents are required to successfully drive a predator away has value in the hope of understanding the effectiveness in making this defensive strategy effective for relevant robotic applications when agent survival is at stake.

\section{References}

[1] Zahavi, A. and Zahavi A., The Handicap principle, Oxford: Oxford University Press. 1997, pg. 125-175

[2] Wright, J. Berg, E. De Kort, S. Khazin, V. and Maklakov, A., "Cooperative sentinel behaviour in the Arabian babbler", Animal Behavior Volume 62, Issue 5, Elsevier, pg. 973-979, 2001

[3] Johnstone, R. and Grafen, A., "Dishonesty and the Handicap Principle", Animal Behavior, Volume 46, pg. 759-764

[4] Lorenz K., On Aggression, New York: Harcourt, Brace \& World Inc.1966, pg. 26

[5] Duncan, B. Ulam, P. and Arkin, R. "Lek Behavior as a Model for Multi-Robot Systems", Proc. IEEE International Symposium on Computational Intelligence in Robotics and Automation (CIRA-09), Daejeon, KR, Dec. 2009.

[6] Tisdale, V. and Fernandez-Juricic, E. "Vigilance and Predator Detection Vary Between Avian Species with Different Visual Acuity and Coverage", Behavioral Ecology Volume 23 pp. 936-945

[7] Wagner, A.R., and Arkin, R.C., 2011. "Acting Deceptively: Providing Robots with the Capacity for Deception", International Journal of Social Robotics, Vol. 3, No. 1, pp. 5-26, 2011.

[8] Bond, C. F., \& Robinson, M., (1988). "The evolution of deception”, Journal of Nonverbal Behavior, 12(4), 295- 307.

[9] U.S. Army (1988.). Field Manual 90-2, Battlefield Deception, http://www.enlisted.info/field- manuals/fm-90-2- battlefielddeception.shtml

[10] Frankenberg, E., "The adaptive significance of avian mobbing 4: Alerting others and perception advertisement in blackbirds facing an owl”, Zeitschrift fur Tierpsychologie, vol. 55, no. 2 pp. 97-118, 1981.

[11] Owings, D. and Coss, R., "Snake Mobbing by California Squirrels: Adaptive Variation and Ontogeny", Behaviour, Vol. 62, No. 1-2 pp. 50-69, 1977.
[12] Balch, T. and Arkin, R.C., "Behavior-based Formation Control for Multi-robot Teams", IEEE Transactions on Robotics and Automation, Vol. 14, No. 6, December 1998, pp. 926-939.

[13] Graw, B. and Manser, M., "The function of mobbing cooperative meerkats", Animal Behaviour, Vol. 73, no. 3 pp. 507-517, 2007

[14] Arkin, R.C., Behavior-based Robotics, MIT Press, 1998.

[15] Wikipedia, accessed 1/21/2012, http://en.wikipedia.org/wiki/File:Redtail_hawk_chased_by_crows_439 1.jpg

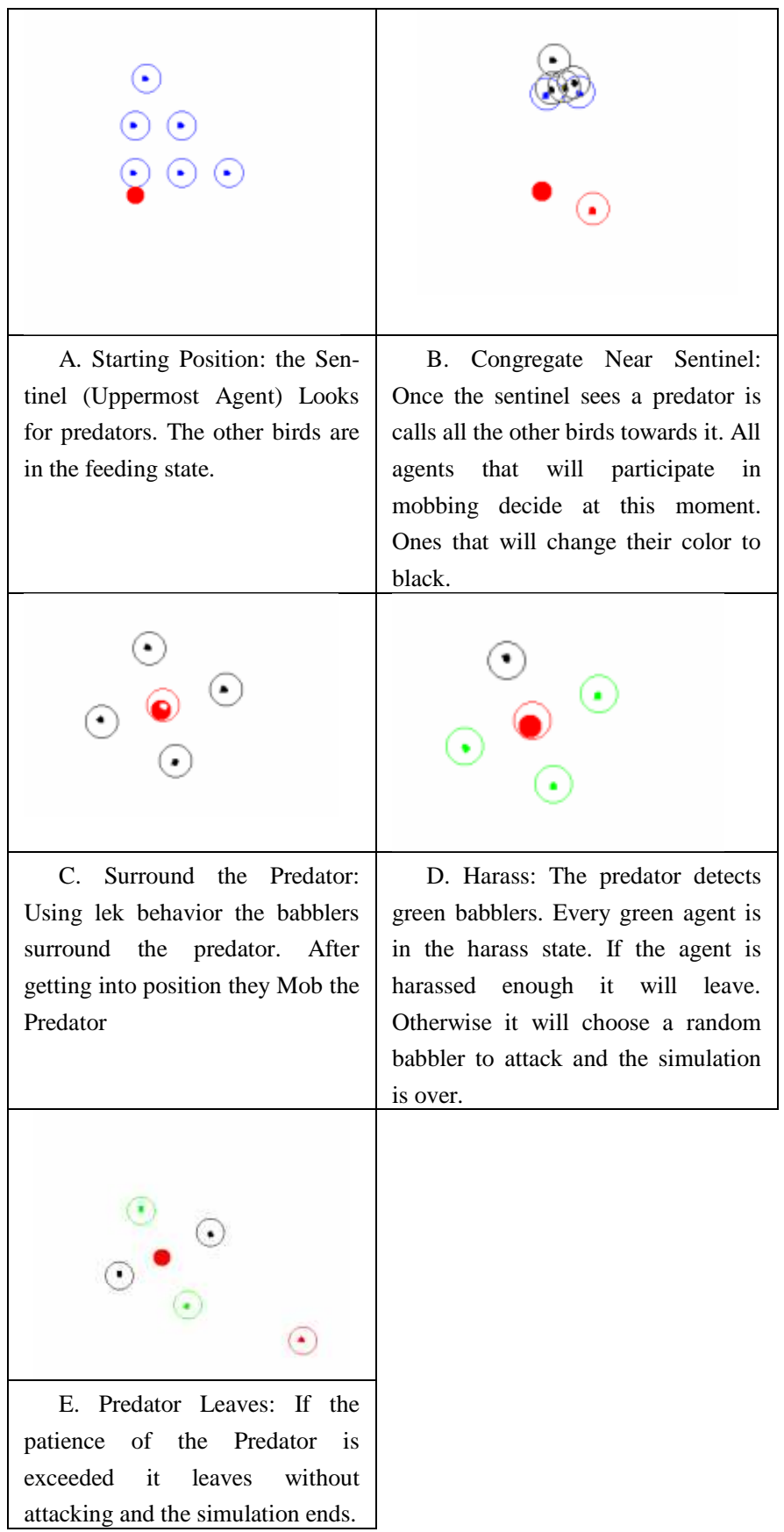

Figure 7: Simulation for Mob Process 


\section{Appendix A: Robot behaviors}

a) Mob: Variable attraction to Predator. Used for approaching predator for mobbing.

$$
V_{\text {mag }}=\frac{H-d}{H}
$$

$V_{\text {direction }}=$ Direction from the center of the robot to the center of the predator

Where:

$\mathrm{d}=$ Distance of robot to predator $\mathrm{H}=$ Maximum predator detection distance

b) Avoid-Obstacle: Repel from object with variable gain and sphere of influence. Used for collision avoidance.

$$
V_{\operatorname{mag}}\left\{\begin{array}{cc}
\infty, & d \leq r \\
\frac{\max -d}{\max -r}, & r<d \leq \max \\
0, & d>\max
\end{array}\right\}
$$

$V_{\text {direction }}=$ Direction from the center of the robot to the center of the obstacle, moving away from obstacle

Where:

$\max =$ Maximum obstacle detection sphere

$d=$ Distance of robot to obstacle

$r=$ Radius of obstacle

c) Noise: Random wander with variable gain and persistence. Used to overcome local maxima, minima, cycles, and for exploration.

$V_{\text {magnitude }}=$ Adjustable gain value

$V_{\text {direction }}=$ Random direction that persists for specified number of steps
Appendix B: Behavioral Assemblages Parameters

\begin{tabular}{|c|c|c|}
\hline Behavior & Parameter & Value \\
\hline \multirow{11}{*}{ 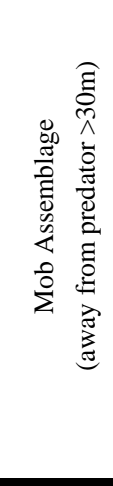 } & Mob Gain & .2 \\
\hline & Noise Gain & .01 \\
\hline & Noise $p$ & 10 \\
\hline & Predator Repel Sphere & $40 \mathrm{~m}$ \\
\hline & Predator Attract Sphere & $50 \mathrm{~m}$ \\
\hline & Predator Detection Sphere & $150 \mathrm{~m}$ \\
\hline & Avoid Obstacle Gain & 1 \\
\hline & Avoid Obstacle Sphere & $70 \mathrm{~m}$ \\
\hline & Avoid Obstacle Safety margin & $2 \mathrm{~m}$ \\
\hline & Predator Perch Spot Gain & .7 \\
\hline & Predator gain & .7 \\
\hline \multirow{11}{*}{ 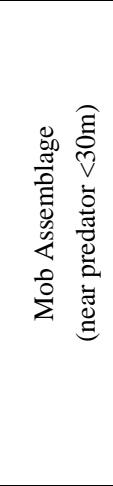 } & Mob Gain & .5 \\
\hline & Noise Gain & .05 \\
\hline & Noise $\mathrm{p}$ & 10 \\
\hline & Predator Repel Sphere & $40 \mathrm{~m}$ \\
\hline & Predator Attract Sphere & $50 \mathrm{~m}$ \\
\hline & Predator Detection Sphere & $150 \mathrm{~m}$ \\
\hline & Avoid Obstacle Gain & .85 \\
\hline & Avoid Obstacle Sphere & $30 \mathrm{~m}$ \\
\hline & Avoid Obstacle Safety margin & $.5 \mathrm{~m}$ \\
\hline & Predator Perch Spot Gain & .5 \\
\hline & Predator gain & 1 \\
\hline
\end{tabular}

Mob Assemblage

\section{Predator}

\begin{tabular}{|c|c|c|}
\hline Behavior/Trigger & Parameter & Value \\
\hline \multirow{11}{*}{ 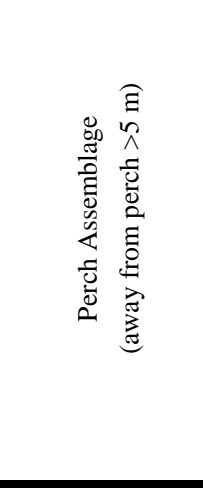 } & Perch Gain & .2 \\
\hline & Noise Gain & .01 \\
\hline & Noise $\mathrm{p}$ & 10 \\
\hline & Predator Repel Sphere & $40 \mathrm{~m}$ \\
\hline & Predator Attract Sphere & $50 \mathrm{~m}$ \\
\hline & Predator Detection Sphere & $150 \mathrm{~m}$ \\
\hline & Avoid Obstacle Gain & .65 \\
\hline & Avoid Obstacle Sphere & $50 \mathrm{~m}$ \\
\hline & Avoid Obstacle Safety margin & $2 \mathrm{~m}$ \\
\hline & Predator Perch Spot Gain & .8 \\
\hline & Predator gain & 0 \\
\hline \multirow{11}{*}{ 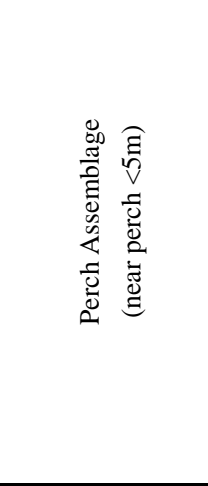 } & Perch Gain & 0 \\
\hline & Noise Gain & .01 \\
\hline & Noise $\mathrm{p}$ & 10 \\
\hline & Predator Repel Sphere & $35 \mathrm{~m}$ \\
\hline & Predator Attract Sphere & $40 \mathrm{~m}$ \\
\hline & Predator Detection Sphere & $300 \mathrm{~m}$ \\
\hline & Avoid Obstacle Gain & .5 \\
\hline & Avoid Obstacle Sphere & $1.4 \mathrm{~m}$ \\
\hline & Avoid Obstacle Safety margin & $.5 \mathrm{~m}$ \\
\hline & Predator Perch Spot Gain & 0 \\
\hline & Predator gain & 0 \\
\hline
\end{tabular}

Perch Assemblage 
Appendix C: Behavioral Assemblages in MissionLab

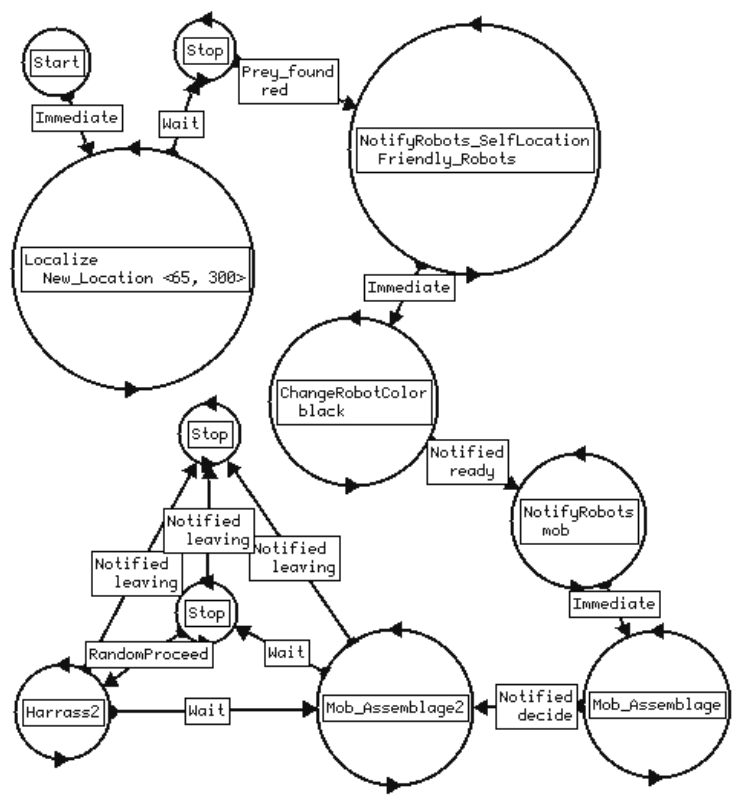

Figure C.1: Cfgedit FSA for the sentinel as implemented in MisisonLab. All communication is done by notification and listening for notifications. It is worth noting that no mobbing occurs until the sentinel notifies all other agents of its location after it detects a red predator. Sub-FSAs include Mob_Assemblage and Harass as explained above. Once the predator attacks or leaves the simulation ends.

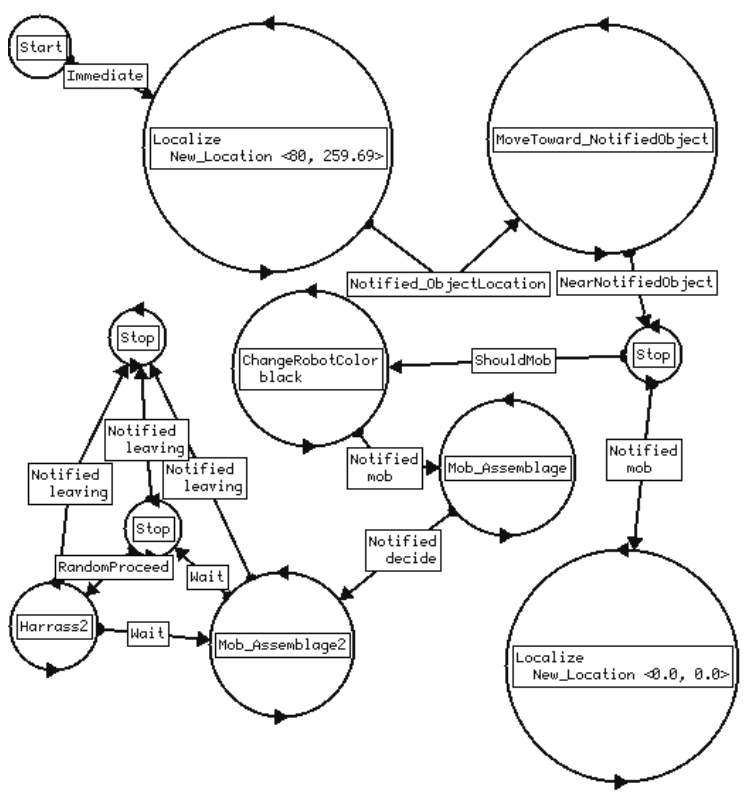

Figure C.2: Cfgedit FSA for the individual babbler as implemented in MisisonLab. Almost identical to sentinel but with a branch for if the ShouldMob trigger is not satisfied. In this case, the babbler moves far away from the group as to not interfere with the simulation. Sub-FSAs are the same as before and the ending conditions are the same.

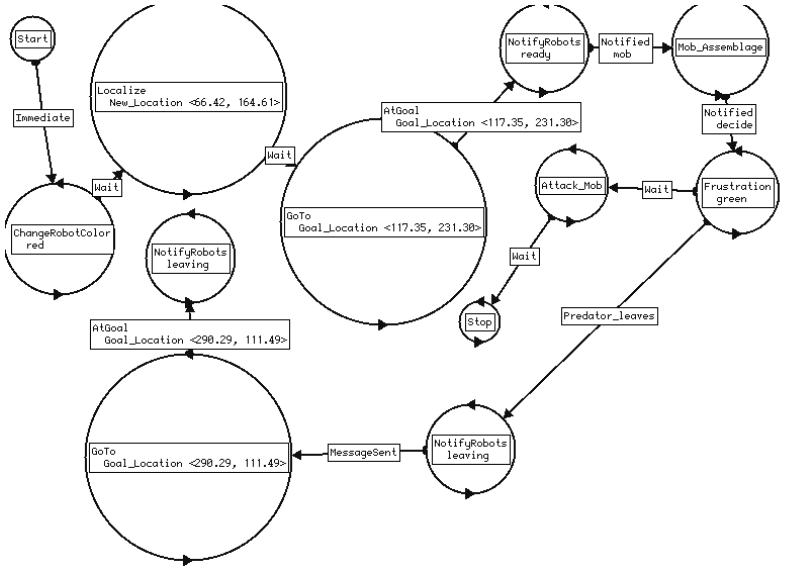

Figure C.3: Cfgedit FSA implemented for the predator. The predator has very little decisions it can make. The only decision is to leave after being frustrated or to attack a member of the mob. The time allotted for this decision is 10 seconds. If frustration threshold is exceeded in this time then the predator attacks a mob member. Similar to the prey, all communication is done by way of message sending. 